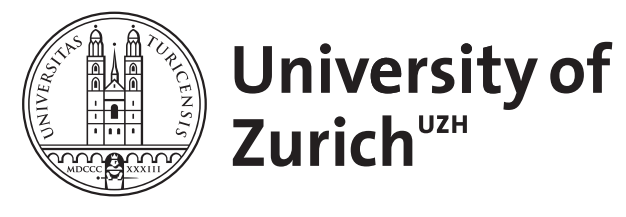

\title{
Wittgenstein und Spengler
}

Ferber, Rafael

\begin{abstract}
In his "Vermischte Bemerkungen", 43, Wittgenstein notices that he was also influenced by Oswald Spengler. The paper deals with the question of in which way Spengler influenced Wittgenstein's late works and if it really was an influence or only a coincidence of ideas. It is put forward that Spengler's rather unknown philosophy of language influenced Wittgenstein's philosophy of language, especially the concepts of family resemblance, antiessentialism, and language-game (Sprachspiel). A picture of the famous Neapolitan gesture of "negation" which motivated Wittgenstein to give up the picture theory of language is also shown with an example taken from Andrea de Jorio, La mimica degli antichi investigata nel gestire napoletano, Napoli 1832.
\end{abstract}

DOI: https://doi.org/10.1515/agph.1991.73.2.188

Posted at the Zurich Open Repository and Archive, University of Zurich

ZORA URL: https://doi.org/10.5167/uzh-70447

Journal Article

Published Version

Originally published at:

Ferber, Rafael (1991). Wittgenstein und Spengler. Archiv für Geschichte der Philosophie, 73(2):188-207. DOI: https://doi.org/10.1515/agph.1991.73.2.188 


\title{
Wittgenstein und Spengler
}

\author{
von Rafael Ferber (Zürich)
}

In den Vermischten Bemerkungen lesen wir: „So haben mich Boltzmann, Hertz, Schopenhauer, Frege, Russell, Kraus, Loos, Weininger, Spengler, Sraffa beeinflußt." $(V B, 43$.$) Wie G. H. v. Wright bemerkt,$ entspricht die Reihenfolge, in der L. Wittgenstein die erwähnten Autoren aufzählt, auch der chronologischen Reihenfolge, in der sie ihn beeinflußt haben. ${ }^{1}$ Mit G. H. v. Wright darf auch bezweifelt werden, ob L. Wittgenstein die Liste später noch fortgesetzt hätte. ${ }^{2} \mathrm{Da} O$. Weininger auf das philosophische Spätwerk keinen ersichtlichen Einfluß mehr ausgeübt $\mathrm{zu}$ haben scheint, ${ }^{3}$ so haben nach $\mathrm{L}$. Wittgensteins Angabe aus dem Jahre 1931 nur zwei Leute Einfluß auf sein Spätwerk genommen, nämlich O. Spengler und P. Sraffa. Den Einfluß P. Sraffas findet man bekanntlich im Vorwort zu den $P U$ neben der Kritik F. Ramseys an Wittgensteins früheren Ideen explizit erwähnt. ${ }^{4}$ Doch worin besteht der Einfluß O. Spenglers auf das Spätwerk? Der Kontext der Bemerkung zeigt, daß O. Spengler nicht nur die „kulturpessimistische " Lebensauffassung L. Wittgensteins beeinflußt hat, ${ }^{5}$ wie sie etwa eine frühere Fassung aus dem Jahre 1930 des Vorwortes zu den Philosophischen Bemerkungen dokumentiert (vgl. $V B, 20-23$ ). Er verdankt ihm auch eine Gedankenbewegung, die er sogleich leidenschaftlich zu seinem Klärungswerk aufgreift:

Das jüdische ,Genie` ist nur ein Heiliger. Der größte jüdische Denker ist nur ein Talent. (Ich z. B.)

Es ist, glaube ich, eine Wahrheit darin, wenn ich denke, daß ich eigentlich in meinem Leben nur reproduktiv bin. Ich glaube, ich habe nie eine Gedankenbewegung erfunden, sondern sie wurde mir immer von jemand anderem gegeben. Ich habe sie nur sogleich leidenschaftlich zu meinem Klärungswerk aufgegriffen. So

1 Wright 1982, 215.

2 Wright 1982, 215.

3 Vgl. zum Einfluß Weiningers auf das Frühwerk Janik 1985, 26-47; Smith 1985, 227-237. Der Einfluß bedarf weiterer Untersuchung.

4 Vgl. dazu den schönen Aufsatz von Lüdeking 1988, 413-416.

5 So als Möglichkeit erwogen von Wright 1982, 215. 
haben mich ... beeinflußt. Kann man als ein Beispiel jüdischer Rèproduktivität Breuer und Freud heranziehen? - Was ich erfinde, sind neue Gleichnisse. (VB, 43. $)^{6}$

Welche Gedankenbewegung ist nun L. Wittgenstein von O. Spengler gegeben und von ihm leidenschaftlich zu seinem Klärungswerk aufgegriffen worden? G. H. v. Wright ist der Ansicht:

Spenglers tatsächlicher Einfluß betrifft anscheinend einen Gedanken in Wittgensteins Spätphilosophie, ja eine ihrer typischsten Gedankenführungen, nämlich den Gedanken der ,Familienähnlichkeiten'. Er entspringt wohl Spenglers Begriff des Ursymbols, das jede der großen Kulturen charakterisiert und das das ausmacht, was Wittgenstein die Familienähnlichkeit zwischen den unterschiedlichen Manifestationen einer Kultur nennt (S. 469): in ihrer Mathematik, Architektur, Religion, in ihrer sozialen und politischen Organisation usw. ${ }^{7}$

Zwar hat meines Wissens O. Spengler noch nicht den Begriff der Familienähnlichkeit zur Charakteristik der Metamorphosen eines Ursymbols in den verschiedenen Manifestationen einer Kultur verwendet. Wir finden höchstens eine Andeutung: „Man vergleiche selbst Goethe oder Raffael mit antiken Menschen, und Heraklit, Sophokles, Plato, Alkibiades, Themistokles, Horaz, Tiberius rücken sofort zu einer einzigen Familie zusammen." $(U A, 148 .)^{8}$ Wenn wir aber den Begriff der Familienähnlichkeit als Begriff L. Wittgensteins zum besseren Verständnis O. Spenglers auffassen, dürfte die Bemerkung G.H. v. Wrights richtig sein: Der Begriff der Familienähnlichkeit ersetzt den des Ursymbols. Zum ersten Mal verwendet nämlich L. Wittgenstein den Begriff in folgendem Kontext:

So könnte Spengler besser verstanden werden, wenn er sagte: ich vergleiche verschiedene Kulturperioden dem Leben von Familien; innerhalb der Familie gibt

6 Vgl. dazu Weininger 1903: „Aus diesem Mangel an Tiefe wird auch klar, weshalb die Juden keine ganz großen Männer hervorbringen können, weshalb dem Judentum, wie dem Weibe, die höchste Genialität versagt ist.“ (424.) „Ein Genie ist Spinoza nicht gewesen". (425.) Doch deswegen ist für Weininger Spinoza noch kein Heiliger, da Heiligkeit und Genialität für ihn zusammengehen: „Alle Genialität ist nur höchste Freiheit vom Naturgesetz. ... Wenn dies so sich verhält, dann ist der Religionsstifter der genialste Mensch. Denn er hat am meisten überwunden.“ (438.) Anscheinend bezieht sich L. Wittgenstein mit „Das jüdische ,Genie' ist nur ein Heiliger." nicht nur auf $O$. Weininger, sondern auch auf $O$. Spengler: „Und ebenso ist die jüdische Mystik, die reiner Sufismus ist, seit den Kreuzzügen dieselbe geblieben, ganz wie die islamische, und sie hat in den letzten Jahrhunderten noch drei Heilige im Sinne des morgenländischen Sufismus hervorgebracht, die man als solche nur erkennt, wenn man durch den Anflug abendländischer Denkformen hindurchzusehen vermag" ( $U A, 956)$, nämlich Spinoza, S. Maimon und O. Weininger (vgl. ebd., $956 \mathrm{f}$.).

.7 Wright 1982, 215. Leider gibt G. H. v. Wright nicht an, nach welcher Ausgabe er den Untergang des Abendlandes zitiert. Mit „S.469“ spielt cr wohl auf dic S. 226 der hier zitierten dtv-Taschenbuchausgabe an.

8 Darauf macht Schulte 1984, 24, aufmerksam.

14 Arch. Gesch. Philosophie Bd. 73 
es cine lamilienähnlichkeit, während es auch zwischen Mitgliedern verschiedener Familien cine Ähnlichkeit gibt; die Familienähnlichkeit unterscheidet sich von der anderen Ähnlichkeit so und so etc. $(V B, 34$.)

R. Haller geht nun einen Schritt weiter. In seinem Aufsatz. War Wittgenstein von Spengler beeinflußt? stellt er die These auf:

Was also war diese Gedankenbewegung? Das ist die Kernfrage und meine Antwort ist: Nicht der Inhalt von Spenglers Analysen, selbst wenn Wittgenstein mit vielen übereingestimmt hätte, sondern ihre Methode; die leitende Idee der Gestaltkunde und Gestaltanalyse der Geschichte.

Die Methode der deskriptiven Morphologie wird der Unangemessenheit der naturwissenschaftlichen Methode für die Behandlung philosophischer Probleme überhaupt entgegengestellt. ${ }^{9}$

Nicht die nomische Naturwissenschaft oder Mathematik mit ihrem „Streben nach Allgemeinheit“ $(B L B, 39)$ bzw. der „verächtlichen Haltung gegenüber dem Einzelfall" $(B L B, 39)$, sondern die vergleichende Morphologie ist das methodische Paradigma der Philosophie, die nicht mehr Allgemeingültigkeit beansprucht (vgl. z. B. $P U, \S 43$ ). Allgemeingültigkeit dagegen ist in der Philosophie meistens der egozentrische Fehlschluß bzw. mit $O$. Spengler zu sprechen:

Es gibt hier [in der lebendigen Welt] nichts Bleibendes und Allgemeines. Man rede nicht mehr von den Formen des Denkens, dem Prinzip des Tragischen, der Aufgabe des Staates. Allgemeingültigkeit ist immer der Fehlschluß von sich auf andere. $(U A, 32$.

Der Antiessentialismus L. Wittgensteins scheint so schon im intendierten Antiessentialismus $O$. Spenglers vorgezeichnet: $O$. Spengler bezeichnet auch den Kern dessen, was er gefunden hat, die vergleichende historische Morphologie nur als „wahr für mich, und, wie ich glaube, auch für die führenden Geister der kommenden Zeit, nicht wahr ,an sich', abgelöst nämlich von den Bedingungen von Blut und Geschichte, denn dergleichen gibt es nicht.“ (UA, VIII.) Die Methode der vergleichenden Morphologie übernimmt O. Spengler aber von Goethe (vgl. UA, IX. 681). Personifiziert ließe sich so sagen: Das Paradigma der Philosophie ist für den späten L. Wittgenstein nicht Newtons Methode der Erklärung durch Gesetze, sondern eher Goethes Methode der Beschreibung von Gestalten. ${ }^{10} \mathrm{DaB}$ aber dieses morphologische Vorbild der philosophischen Methode auf den unmittelbaren Einfluß O. Spenglers zurückgeht, wird von R. Haller überzeugend dargelegt. Die Überlegungen R. Hallers lassen sich durch ein Zeugnis aus den Bemerkungen über Frazers Golden Bough ergänzen:

Der Begriff der übersichtlichen Darstellung ist für uns von grundlegender Bedeutung. Er bezeichnet unsere Darstellungsform, die Art, wie wir die ${ }_{6}$ Dinge sehen. (Eine Art der ,Weltanschauung', wie sie scheinbar für unsere Zeit typisch ist. Spengler.) $(B F G B, \cdot 37$.

9 Haller, 1982; 176.

10 Vgl. dazu Schulte 1984, 1-32. 
Die „übersichtliche Darstellung“ wird in den $P U$ durch die Sprachspiele und die Familienähnlichkeiten zwischen den Spielen geleistet. Diese „übersichtliche Darstellung" bezieht L. Wittgenstein als eine „Art von ,Weltanschauung', wie sie scheinbar für unsere Zeit typisch ist“, auf O. Spengler zurück. „Übersichtliche Darstellung“ aber heißt in der Sprache O. Spenglers eine Darstellung mittels einer vergleichenden Morphologie der Kulturformen. Für L. Wittgenstein wäre es eine vergleichende Morphologie der Sprachformen: „Was ich angebe, ist die Morphologie des Gebrauchs eines Ausdrucks.“" stein von O. Spengler, der seine Methode der vergleichenden Morphologie auch auf die philosophischen Fragen ausgedehnt haben möchte. Die von G.P. Baker und P. M.S. Hacker nicht identifizierte Stelle lautet:

..., daß jede philosophische Frage nur der verhüllte Wunsch ist, eine bestimmte Antwort zu erhalten, die in der Frage schon beschlossen liegt, daß man die großen Fragen einer Zeit gar nicht vergänglich genug fassen kann und daß demnach eine Gruppe historisch bedingter Lösungen angenommen werden muß, deren $\ddot{U}$ bersicht erst - unter Ausschaltung aller eigenen Wertmaßstäbe - die letzten Geheimnisse aufschließt. $(U A, 34$.

Der Gedanke aus den Bemerkungen über Frazers Golden Bough taucht aber im Paragraphen 122 der $P U$ unter Weglassung von O. Spenglers Namen und mit der vorsichtigen Frage „Ist dies eine ,Weltanschauung'?“ wieder auf:

Der Begriff der übersichtlichen Darstellung ist für uns von grundlegender Bedeutung. Er bezeichnet unsere Darstellungsform, die Art, wie wir die Dinge sehen. (Ist dies eine ,Weltanschauung'? ${ }^{12}$

Diese quasispenglersche „Weltanschauung“ L. Wittgensteins scheint sich aber nicht nur auf die Methode, sondern auch auf den „Inhalt von Spenglers Analysen“ zu beziehen, mit denen L. Wittgenstein im Gegensatz zum Irrealis R. Hallers ,selbst wenn Wittgenstein mit vielen übereingestimmt hätte, ..." (Hervorhebung R. F.) wenigstens hinsichtlich der Sprachphilosophie zumindest partiell übereingestimmt hat. Ich entwickle diese methodische und inhaltliche Übereinstimmung in drei Schritten. Abschließend möchte ich der Frage nachgehen, ob es sich hier nur um eine Koinzidenz O. Spenglers und L. Wittgensteins oder auch um einen Einfluß O. Spenglers auf L. Wittgenstein handelt.

11 Malcolm 1987, 72.

12 Vgl. zu „Übersicht“ das informative Kapitel in Baker and Hacker 1980, 531 545. Die Exegese von $\S 122$ läßt den Hinweis auf Spengler, $U A, 34$, vermissen. Mit Weltanschauung dürfte aufgrund dieser textlichen Evidenz O. Spengles "Weltanschauung“ gemeint sein, die „scheinbar für unsere Zeit typisch ist". Dic Folgerung Baker/Hackers, 1980: „Nevertheless, it [this kind of Weltanschauung] is particularly necessary in our times“, 547, wird durch den Text nicht gestützt. 
I.

DaB O. Spengler eine Sprachphilosophie entwickelt hat, scheint nicht nur Sprachphilosophen und Linguisten, sondern auch Wittgensteinforschern völlig unbekannt. Auch in den nach den Vermischten Bemerkunge'n (1977) publizierten wichtigeren Arbeiten zu L. Wittgenstein wie etwa denjenigen G. Bakers und P. M. Hackers, S. A. Kripkes, D. Bloors, C. McGinns, J. Schultes, J. und M. Hintikkas, N. Malcolms, S. Stephen Hilmys, E. v. Savignys, D. Pears, B. McGuiness' finden wir wie in R. Hallers Aufsatz keinen Hinweis auf O. Spenglers Sprachphilosophie. Der einzige mir bekannte Hinweis ist eine allerdings irreführende Fußnote G.H. v. Wrights und zwar nur in der deutschen Übersetzung seines Aufsatzes Wittgenstein und seine Zeit. ${ }^{13}$ Die Klage O. Spenglers

13 Vgl. Baker/Hacker 1980; Kripke 1982, Bloor 1983; McGinn 1984; Schulte 1984; Haller 1986; Hintikka 1987; Malcolm 1987; Hilmy 1987; Savigny 1988; Pears 1988; McGuiness 1988. Hinweise auf Spengler enthalten Baker/Hacker 1980, 136, 470 n., 483 n., 538, 546-548, 556; Bloor 1983, 31, 32, 162-167, 175, 204; Schulte 1984, 23 f; Haller 1986, 170-186; Hilmy 1987, 83-86, 96, 114, 189, Anm. 228, 230, 428, 432, 494; McGuiness 1988, 68, 74, 145. Die ausführlichsten Hinweise bietet außer Haller 1986, 170-186; Hilmy 1987, insb. Anm. 230, Anm. 428, Anm. 432. Keiner dieser Autoren verweist auf Spenglers Sprachphilosophie. Hilmys Hypothese, Anm. 230, der Begriff der Familienähnlichkeit entstamme in Reaktion auf folgende Stelle: „Aber ,die Menschheit' hat kein Ziel, keine Idee, keinen Plan, so wenig wie die Gattung der Schmetterlinge oder der Orchideen ein Ziel hat" $(U A, 28)$, scheint zu wenig fundiert. McGuiness 1988, 68, erwähnt nur einen Einklang L. Wittgensteins mit der Spenglerschen Auffassung „..., die abendländische Kultur sei nichts weiter als eine von mehreren Phasen der Menschheitsgeschichte, ...“. Auf derselben Seite aber schreibt McGuiness: „Auf die großen Dichter - Goethe, Schiller, Mörike, Lessing - kommt er sein Leben lang immer wieder zurück. Sie seien wie Sonnen, sagt er, womit er meint, daß sie im Mittelpunkt einer Kultur stehen und diese bestimmen“. Wie McGuiness nicht zu sehen scheint, wandelt hier Wittgenstein in einer für seine Spenglerrezeption vielleicht prototypischen Art und Weise eine Metapher O. Spenglers aus der Einleitung in den Untergang des Abendlandes ab: „Man wählt eine einzelne Landschaft zum natürlichen Mittelpunkt eines historischen Systems. Hier ist die Zentralsonne. Von hier aus erhalten alle Ereignisse der Geschichte ihr wahres Licht. Von hier aus wird ihre Bedeutung perspektisch abgemessen“. $(U A, 23$.) Die einzige mir bekannte Ausnahme ist eine Fußnote bei Wright 1982, 215: „Es bestehen Ähnlichkeiten zwischen Spenglers Sprachauffassung - avor allem in seinem späteren Buch Der Mensch und die Technik,1931 - und Wittgensteins Sprachkonzeption im Braunen Buch und zu Beginn der Untersuchungen. Auf diese Ähnlichkeiten, die ich beachtenswert finde, bin ich von H.J. Dahms hingewiesen worden. Dagegen sind Spenglers in dem genannten Werk vorgetragene Theorien über den Ursprung der Sprache und den Zweck der sprachlichen Verständigung , unwittgensteinianisch““. Sowohl über den Ursprung der Sprache 
in den unter dem Titel Frühzeit der Weltgeschichte aus dem Nachlaß publizierten Fragmenten scheint so noch heute nicht ganz unberechtigt:

Was ich [im] ,Untergang des Abendlandes' II über Rasse, Völker [und] Sprachen gesagt habe, ist unverstanden und unbeachtet geblieben. Hätte man es durchdacht, so wäre man weiter. ${ }^{14}$

O. Spengler hat nämlich unter dem Titel Völker, Rassen, Sprachen im Zweiten Kapitel: Städte und Völker des Zweiten Bandes: Welthistorische Perspektiven seines Hauptwerkes eine eigentümliche Sprachphilosophie publiziert. Da sie mit ihrem morphologischen Entwicklungsschema der Sprache von Vorkultur über Kultur zur Zivilisation einen Neuansatz markiert, so dürfte es zwar überraschend, aber korrekt sein, O. Spengler nicht nur zu den Geschichtsphilosophen zu zählen, sondern auch in die Reihe der deutschen Sprachphilosophen wie J.G. Herder, J.G. Hamann, W. v. Humboldt und andere einzuordnen. In einem in seiner morphologischen Methode schauender Physiognomik (vgl. UA, 125152) fundierten Vollzug der Maxime „.... denk nicht, sondern schau!“ $(P U, \S 66)$ geht $O$. Spengler von folgendem „Urphänomen“ sprachlicher Verständigung aus:

Wer in das Wesen der Sprache eindringen will, der lasse alle gelehrten Wortuntersuchungen beseite und beobachte, wie ein Jäger mit seinem Hunde spricht. Der Hund folgt dem ausgestreckten Finger, er horcht angespannt auf die Wortklänge und schüttelt dann den Kopf; er versteht diese Art Menschensprache nicht. Dann macht er ein paar Sätze, um seine Auffassung anzudeuten, bleibt stehen und bellt: das ist ein Satz in seiner Sprache, der die Frage enthält, ob der Herr etwa dies gemeint hat. Dann folgt, ebenfalls in einer Hundesprache ausgedrückt, die Freude, wenn er begreift, daß er recht hatte. Genau so versuchen sich zwei Menschen zu verständigen, die keine einzige Wortsprache wirklich gemein haben. Wenn ein Landpfarrer einer Bäuerin etwas erklärt, so sieht er sie scharf an und unwillkürlich legt er alles in seine Gebärde, was sie in der kirchlichen Ausdrucksweise ja doch nicht verstehen würde. Die heutigen Wortsprachen können sämtlich nur in Verbindung mit anderen .Spracharten zur Verständigung führen. Für sich allein sind sie nie und nirgends in Gebrauch gewesen. $(U A, 713$.

Die „Urszene“ der $P U$ lautet bekanntlich:

Denke nun an diese Verwendung der Sprache: Ich schicke jemand einkaufen. Ich gebe ihm einen Zettel, auf diesem stehen die Zeichen: ,fünf rote Äpfel‘. Er trägt

als auch über den Zweck der sprachlichen Verständigung bestehen aber auch in Der Mensch und die Technik Âhnlichkeiten mit der Auffassung Wittgensteins. Dieses Werk erläutert nur, was der $U A$ ausführlicher dargestellt hat, vgl. Spengler 1931, 40.

14 Spengler 1966, 137. 
den Zellel aum Kaufmann; der öffnet die Lade, auf welcher das Zeichen, Äpfel steht; dann sucht er in einer Tabelle das Wort ,rot' auf und findet ihm gegenüber ein Farbmuster; nun sagt er die Reihe der Grundzahlwörter - ich nehme an, cr weib sie auswendig - bis zum Worte ,fün' und bei jedem Zahlwort nimmt er einen Apfel aus der Lade, der die Farbe des Musters hat. - So, und ähnlich, operiert man mit Worten. - ,Wie weiß er aber, wo und wie er das Wort ,rot nachschlagen soll und was er mit dem Wort ,fünf ' anzufangen hat?" - Nun, ich nehme an, er handelt, wie ich es beschrieben habe. Die Erklärungen haben irgendwo ein Ende. - Was ist aber die Bedeutung des Wortes ,fünf"? - Von einer solchen war hier gar nicht die Rede; nur davon, wie das Wort ,fünf gebraucht wird. $(P U, \S 1$.)

Gemeinsam ist beiden fingierten Urszenen, daß sie paradigmatische Beispiele sprachlicher Kommunikation sind. Paradigmatisch ist die Szene für O. Spengler, da er in diesem einen Beispiel die Urgestalt oder das Urphänomen sprachlicher Verständigung sieht, das in viel komplizierteren und höheren Formen wiederkehrt. Paradigmatisch ist die Szene für L. Wittgenstein, weil sie mutatis mutandis in den Sprachspielen der Paragraphen 2 und 8 wiederauftaucht und diese Sprachspiele als „Vergleichsobjekte“ dastehen, „die durch Ähnlichkeit oder Unähnlichkeit ein Licht in die Verhältnisse unserer Sprache werfen sollen" $(P U, \S 130)$. Es handelt sich um paradigmatische Beispiele sprachlicher Kommunikation, weil beide nicht monologisch, sondern dialogisch erfolgen. Dies ist für O. Spengler entscheidend, wie dann aus dem Kapitel Die zweite Stufe: Sprechen und Unternehmen von Der Mensch und die Technik explizit hervorgeht:

Das Sprechen erfolgt nicht monologisch, sondern dialogisch, die Satzreihen folgen nicht als Rede, sondern zwischen mehreren Menschen als Unterredung. Der Zweck ist nicht ein Verstehen aus dem Nachdenken heraus, sondern eine wechselweitige Verständigung durch Frage und Antwort. ${ }^{15}$

$\mathrm{Da}$ diese primitiven Sprachspiele bei L. Wittgenstein nicht monologisch, sondern dialogisch gestaltet sind (vgl. $P U, \S 1,2,8)$, ist denn auch bei L. Wittgenstein kein Zufall. Es ist u. a. darin begründet, daß es keine Privatsprache gibt, die nicht durch eine öffentliche Sprache definiert wäre, insofern man nicht privatim einer Regel folgen kann (vgl. $P U, \S 202$ ). Doch dieser methodische Ausgang von einem paradigmatischen dialogischen Beispiel ist nur eine formale Gemeinsamkeit. Entscheidender scheint die inhaltliche Übereinstimmung, daß sowohl

15 Spengler 1931, 42. 
für O. Spengler wie auch für L. Wittgenstein die Wortsprache in körperliche Handlungen eingebettet ist: Die an den Hund gerichteten Wortklänge des Jägers werden begleitet vom Ausstrecken des Fingers. Auch im ersten Beispiel L. Wittgensteins verläuft die Wortsprache „fünf rote $\ddot{A p f e l “ ~ u n d ~ d a s ~ A u f z a ̈ h l e n ~ d e r ~ f u ̈ n f ~ Z a h l w o ̈ r t e r ~ d u r c h ~ d e n ~}$ Kaufmann - im Fluß entsprechender Handlungen: Der Käufer trägt den Zettel zum Kaufmann, der öffnet die Lade, sucht in einer Tabelle das Wort „rot“ heraus, nimmt bei jedem Zahlwort einen Apfel aus der Lade, der die Farbe des Musters hat, ... - .., ,er handelt, wie ich es beschrieben habe“. Ebenso handelt der Hund auf den ausgestreckten Finger und die Wortklänge des Jägers hin: Er schüttelt den Kopf, macht ein paar Sätze usw. O. Spengler führt dieses Beispiel dahingehend aus, daß er den Begriff der Sprache auf das körperliche kommunikative Handeln ausdehnt:

Diese Sprache reden wir heute noch sämtlich, ohne es zu wissen. Das Kind spricht lange bevor es das erste Wort gelernt hat, und die Erwachsenen sprechen mit ihm, ohne irgendwie an die gewohnte Wortbedeutung zu denken; das heißt, die Lautgebilde dienen hier einer ganz anderen als der.Wortsprache. Auch diese Sprachen haben ihre Gruppen und Dialekte; sie können gelernt, beherrscht und mißverstanden werden; sie sind für uns so unentbehrlich, daß die Wortsprache den Dienst versagen würde, wenn wir je den Versuch machten, sie für sich allein, ohne Ergänzung durch Ton- und Gebärdensprache anzuwenden. ( $U A, 713 \mathrm{f}$.)

O. Spengler geht so weit zu behaupten, daß es ein Grundfehler der Sprachwissenschaft ist, daß sie Sprache überhaupt und menschliche Wortsprache verwechselt - nicht theoretisch, aber regelmäßig in der Praxis aller Untersuchungen (vgl. UA, 714). Das habe ,zu einer maßlosen Unkenntnis der unübersehbaren Menge von Spracharten geführt, die unter Tieren und Menschen im allgemeinen Gebrauch sind" ( $U A$, 714). Er schließt den Abschnitt mit der Pointe: „Mit dem Menschen darf eine Untersuchung der Sprache sicherlich nicht beginnen" (UA, 714). - Nun beginnt zwar L. Wittgenstein die Untersuchung der Sprache in den $P U$ mit dem Menschen. Doch ist für ihn das Sprachspiel keineswegs nur ein Wortspiel, vielmehr: „Ich werde auch das Ganze: der Sprache und der Tätigkeiten, mit denen sie verwoben ist, das ,Sprachspiel“ nennen“ $(P U, \S 7)$. „Spiel“" hat hier keine ludistische Konnotation, sondern die von Tätigkeit: „Das Wort ,Sprachspiel" soll hier hervorheben, daß das Sprechen der Sprache ein Teil ist einer Tätigkeit, oder einer Lebensform“" $(P U, \S 23)$. Insoweit behält auch L. Wittgenstein 
noch das Augustinische Bild von der Gebärdensprache als ,der natürlielien Sprache aller Völker" in seinem Begriff des Sprachspiels bei (vgl. $P U, \$ 7, \$ 206)$. In den Zetleln lesen wir gar in etwas anderem, nämlich auf das Schmerzverhalten zentriertem Kontext: „Unser Sprachspiel ist ein Ausbau des primitiven Benehmens. (Denn unser Sprachspiel ist Benehmen.) (Instinkt.)“ $(Z, \S 545)$. Noch deutlicher geht diese Auffassung der Sprache als „Ausbau primitiven Benehmens" aus der grundlegenden Bemerkung in Über Gewißheit hervor.

Ich will den Menschen hier als Tier betrachten; als ein primitives Wesen, dem man zwar Instinkt, aber nicht Raisonnement zutraut. Als ein Wesen in einem primitiven Zustande. Denn welche Logik für ein primitives Verständigungsmittel genügt, deren brauchen wir uns auch nicht zu schämen. Die Sprache ist nicht aus einem Raisonnement hervorgegangen. ( $U G, \S 475$.)

Der letzte Satz könnte fast aus der Feder O. Spenglers stammen. Wir lesen in Der Mensch und die Technik:

Der ursprüngliche Zweck des Sprechens ist die Durchführung einer Tat nach Absicht, Zeit, Ort, Mitteln. Die klare, eindeutige Fassung derselben ist das Erste, und aus der Schwierigkeit, sich verständlich zu machen, den eigenen Willen anderen aufzuerlegen, ergibt sich die Technik der Grammatik, die Technik der Bildung von Sätzen und Satzarten, des richtigen Befehlens, Fragens, Antwortens, der Ausbildung von Wortklassen aufgrund der praktischen, nicht der theoretischen Absichten und Ziele. Das theoretische Nachdenken hat am Entstehen des Sprechens in Sätzen so gut wie gar keinen Anteil. Alles Sprechen ist praktischer Natur und geht vom ,Denken der Hand aus. ${ }^{16}$

Sprechen ist wie die Hand ein Mittel, welches das "Tun zu mehreren““ ermöglicht. Das heißt: Sprechen ist wie das mit der Hand entstandene Werkzeug multifunktional. O. Spengler scheint hier mit dem Satz „Alles Sprechen ist praktischer Natur und geht vom ,Denken der Hand" aus“ den Begriff der Sprechhandlung zu antizipieren, wiewohl der Ausdruck dafür allerdings meines Wissens erstmals in der Philosophischen Grammatik L. Wittgensteins die „Sprechschwelle“ übertritt: „Die Sprache ist für uns ein Kalkül; sie ist durch die Sprachhandlungen charakterisiert" ( $P G$, 193). „Alles Sprechen ist praktischer Natur und geht vom ,Denken'der Hand" aus" heißt: Alles Sprechen ist handelnder Natur und geht vom „Denken der Hand“, dem „Tun zu mehreren“" (Hervorhebung R. F.) aus.

16 Spengler $1931,43$. 
II.

Doch wenn Sprechen ein Tun ist und mehreres tut, was tut es ursprünglich? O. Spenglers Urphänomen oder Ursymbol des Jägers, der mit ausgestrecktem Finger mit seinem Hunde spricht, deutet auf einen Befehl an den Hund hin. Den Eindruck bestätigt die Spenglersche Hypothese, daß bei Mikroorganismen nicht nur ein Wille zum Empfangen von Eindrücken - Orientierung -, sondern auch ein Wille zum Erzeugen von Eindrücken bei anderen vorhanden sei: sie sollen angelockt, erschreckt, verjagt werden (vgl. $U A, 715$ ):

Dies nennen wir Ausdruck und mit ihm ist das Sprechen als Tätigkeit des tierischen Wachseins gegeben. Seitdem ist nichts grundsätzlich Neues hinzugekommen. Die Weltsprachen hoher Zivilisationen sind nichts als äußerst verfeinerte Ausgestaltungen von Möglichkeiten, welche sämtlich schon in der Tatsache des gewollten Eindrucks einzelliger Wesen aufeinander enthalten sind. ( $U A, 715$.)

„Sprechen als Tätigkeit des tierischen Wachseins“ gehört offensichtlich in die Naturgeschichte. Insoweit sogar die Weltsprachen hoher Zivilisationen nichts anderes als äußerst verfeinerte Ausgestaltungen solch biologischer Möglichkeiten sind, so gehören sie nicht weniger dazu. Es gibt insoweit keinen Bruch zwischen tierischer und menschlicher Sprache. Die Sprachgeschichte verläuft für O. Spengler „an sich im biologischen Weltbild“ ( $U A, 731)$. Er antizipiert dabei, wenn auch ohne L. Wittgensteins Begründung, etwas Ähnliches wie Wittgensteins Einsicht „Befehlen, fragen, erzählen, plauschen gehören $\mathrm{zu}$ unserer Naturgeschichte so wie gehen, essen, trinken, spielen“ $(P U, \S 25) .{ }^{17}$ Die genetisch ursprüngliche Sprechhandlung ist dabei für $O$. Spengler keineswegs die deskriptive, sondern die direktive. Dies geht noch deutlicher aus den Erläuterungen seiner Sprachphilosophie in Der Mensch und die Technik hervor. Er kritisiert dort nicht nur den Grundfehler der Romantiker, die Sprache aus einer „Urpoesie der Menschheit" abzuleiten, sondern auch - wie schon im Untergang des Abendlandes (vgl. $726 \mathrm{f}$.) -18 den „Grundfehler der Feinde aller Romantik, der Rationalisten": 19

17 Vgl. dazu den Kommentar von Savigny 1988, 60, ad loc.

18 Vgl. insbesondere die Aussage: „Aber nach der gewöhnlichen Definition ist der

- Satz der sprachliche Ausdruck eines Gedankens, nach H. Paul ein Symbol für die Verbindung mehrerer Vorstellungen in der Seele des Sprechenden. Alle diese Bestimmungen widersprechen sich." $(U A, 726$.

19 Spengler 1931, 41. 
Sie laulen stets der Meinung nach, daß der Satz cin Urteil oder einen Gedunke'n ausdrücke. Sie sitzen an ihrem Schreibtisch voller Bücher und grübeln über ihr eigenes Denken und Schreiben nach. Da scheint ihnen der, Gedanke' der Zweck des Sprechens zu sein. Weil sie allein zu sitzen pflegen, vergessen sie über dem Sprechen das Hören. Über der Frage die Antwort, über dem Ich das $D u$. Sie sagen ,Sprache und meinen die Rede, den Vortrag, die Abhandlung. Ihre Ansicht vom Entstehen der Sprache ist monologisch und deshalb falsch. ${ }^{20}$

Gegen diese „rationalistische“ Auffassung antwortet O. Spengler in Der Mensch und die Technik auf seine Frage: „Welches sind die ursprünglichen Formen des Sprechens?“:

Nicht das Urteil, die Aussage, sondern der Befehl, der Ausdruck des Gehorsams, die Feststellung, die Frage, die Bejahung, die Verneinung. Es sind Sätze, die sich stets an einen anderen wenden, ursprünglich sicher ganz kurz: Tu das! Fertig? Ja! Anfangen! ${ }^{21}$

Ein von O. Spengler unbekannterweise bekämpfter „monologischer Rationalist" war wohl auch sein späterer „Gefolgsmann“, der Verfasser des Tractatus: „Im Satz drückt sich der Gedanke sinnlich wahrnehmbar aus" (TLP, 3.1). Es brauchte die Arbeit L. Wittgensteins "An der eignen Auffassung“ (vgl. VB, 38), um sich von der Ansicht zu befreien, daß jeder Satz einen Gedanken ausdrücken müsse; einen Kampf, den er insbesondere bei Sätzen mit Verben der ersten Person Singular über mentale Zustände geführt hat. Er findet sich in den Philosophischen Bemerkungen und im Braunen Buch dokumentiert. N. Malcolm hat ihn im ausgezeichneten Kapitel Language as Expressive Behaviour von Nothing Is Hidden nachgezeichnet, allerdings ohne die verborgenen Ähnlichkeiten mit O. Spenglers analogem Kapitel (vgl. UA, 712-721) auch nur mit einem Worte zu erwähnen. ${ }^{22}$ Signifikanterweise sind die primitiven Sprachspiele der Paragraphen 1, 2, 8 der PU Sprachen, die nur aus Befehlen bestehen (vgl. $P U, \S 18$ ). In der Aufzählung des Paragraphen 25 „Befehlen, fragen, erzählen, plauschen ...“ findet sich das Befehlen, in der "Mannigfaltigkeit der Sprachspiele“ des Paragraphen 23 das Befehlsspiel an erster Stelle: „Befehlen, und nach Befehlen handeln - “ $(P U, \S 23)$. Ferner ist einer Regel folgen analog dem: „einen Befehl befolgen“ $(P U, \S 206)$. Die prototypische Form des Spre-

20 Spengler 1931, 41.

21 Spengler 1931, 42f.

22 Vgl. Malcolm 1987, 133-153. 
chens scheint so der Befehl. Das heißt nicht, daß für L. Wittgenstein solche Befehlsspiele wie für $\mathrm{O}$. Spengler auch genetisch ursprünglich sind, wohl aber daß sie - ähnlich wie das Urphänomen des Jägers mit dem Hund bei O. Spengler - als die primären "Vergleichsobjekte“ dastehen, „die durch Ähnlichkeit und Unähnlichkeit ein Licht in die Verhältnisse unsrer Sprache werfen sollen“ $(P U, \S 130)$. Und wie $\mathrm{O}$. Spengler - wenn auch aus besseren Gründen - bricht L. Wittgenstein mit der „rationalistischen“ Idee, „die Sprache funktioniere immer auf eine Weise, diene immer dem gleichen Zweck: Gedanken zu übertragen - seien dies nun Gedanken über Häuser, Schmerzen, Gut und Böse, oder was immer" $(P U, \S 304)$.

\section{III.}

Wie steht es aber mit Name und Satz in der Sprachphilosophie des Untergangs? In der Geschichte der Wortsprachen unterscheidet $\mathrm{O}$. Spengler drei Phasen. Sie entsprechen Vorzeit, Kultur und Zivilisation in der Geschichte der Kulturen:

Auf der ersten erscheinen innerhalb hochentwickelter, aber wortloser Mitteilungssprachen die ersten Namen als Größen eines neuartigen Verstehens. Die Welt erwacht als Geheimnis. Das religiöse Denken beginnt. Auf der zweiten wird nach und nach eine vollständige Mitteilungssprache in grammatische Werte umgesetzt. Die Geste wird zum Satz und der Satz verwandelt die Namen in Worte. $(U A, 731$.)

Die dritte Stufe wird durch einen raschen Flexionsverfall und damit „den Ersatz der Grammatik durch die $\operatorname{Syntax}(U A, 731)$ bezeichnet. Dies ist die Stufe der Zivilisation, in der erst die Schriftsprache entsteht (vgl. $U A, 731$ f.). Die Frage, ob die wortlosen Mitteilungssprachen schon vor dem Auftreten echter Namen wirkliche „Sätze“ enthielten, scheint O. Spengler müßig. Der Satz im heutigen Sinne setze das Dasein der Namen voraus (vgl. $U A, 725 \mathrm{f}$.):

Der Name tritt als etwas Neues ganz für sich auf. Die Wortarten aber entstehen als Satzelemente; und nun strömen in unübersehbarer Menge die Wachseinsinhalte heran, die bezeichnet, in dieser Welt von Worten vertreten sein wollen, bis zuletzt, alles' für das Nachdenken in irgendeiner Weise Wort geworden ist. (UA, 726.)

Zwischen Satz und Wort wird dabei nicht etwa aufgrund des Inhalts unterschieden: 
Aber nach der gewöhnlichen Definition ist der Satz der sprachliche Ausdruck cines Gedankens, nach H. Paul ein Symbol für die Verbindung mehrerer Vorstellungen in der Seele des Sprechenden. Alle diese Bestimmungen widersprechen sich. Es scheint mir ganz unmöglich, das Wesen des Satzes aus dem Inhalt zu ermitteln. Tatsache ist lediglich, daß wir die relativ größten mechanischen Einheiten im Sprachgebrauch Sätze, die relativ kleinsten Worte nennen. $(U A, 726$.

Im Sinne des späten L. Wittgenstein ist wohl die am Sprachgebrauch orientierte, flexible Unterscheidung zwischen Satz und Wort, wie sie der Paragraph 19 der $P U$ weiterentwickelt. Im Sinne des späten L. Wittgenstein dürfte ebenfalls die These sein, daß das Sprechen in Worten „etwas sehr Spätes und Abgezweigtes, eine letzte Blüte am Stamme der Lautsprachen" $(U A, 722)$ darstelle, wie das O. Spengler gegen die Sprachtheorien W. Wundts und J. O. Jespersens moniert:

Es ist der gleiche Fehler so entgegengesetzter Theorien wie derjenigen Wundts und Jespersens, daß sie das Sprechen in Worten wie etwas ganz Neues und Fürsichseiendes untersuchen, was notwendig zu einer durchaus falschen Deutung führt. $(U A, 721-722$.)

Nicht mehr im Sinne des späten L. Wittgenstein dürfte allerdings die These $O$. Spenglers sein, daß die Namen nur durch eine „tiefe Verwandlung der Seele" $(U A, 723)$ entstanden sind:

Man kann sich die ursprüngliche Namengebung nicht feierlich und ehrfürchtig genug denken. Man soll den Namen nicht immer nennen, man soll ihn geheim halten, es liegt eine gefährliche Macht in ihm. Mit dem Namen ist der Schritt von der alltäglichen Physik des Tieres zur Metaphysik des Menschen vollzogen. Es war die größte Wendung in der Geschichte der menschlichen Seele. Die Erkenntnistheorie pflegt Sprache und Denken nebeneinander zu stellen, und wenn man allein die heute noch erreichbaren Sprachen beachtet, trifft das zu. Ich glaube aber, daß man viel tiefer greifen kann: mit dem Namen ist die eigentliche, bestimmte Religion innerhalb einer formlosen, allgemein religiösen Scheu entstanden. Religion in diesem Sinne heißt religiöses Denken. - Von diesem religiösen Urdenken ist alles philosophische, gelehrte, wissenschaftliche Denken später Zeiten bis in seine letzten Gründe abhängig geblieben. (UA, 723-724.)

Zwar schreibt L. Wittgenstein noch in den Bemerkungen über Frazers Golden Bough:

Warum sollte dem Menschen sein Name nicht heilig sein können. Ist er doch einerseits das wichtigste Instrument, das ihm gegeben 
wird, anderseits wie ein Schmuckstück, das ihm bei der Geburt umgehangen wird. (BFGB, 33.)

Doch ist die Spenglersche Auffassung der ursprünglichen Namengebung nicht nur mentalistisch durchtränkt, sondern sie scheint auch als ein paradigmatisches Beispiel jener Auffassung des Benennens als ,irgend ein[es] merkwürdige[n] seelische[n] Akt[es], quasi eine[r] Taufe eines Gegenstandes“ $(P U, \S 38)$, die L. Wittgenstein im Paragraphen 38 der $P U$ und anderswo bekämpft. Hier trennen sich offensichtlich die Wege L. Wittgensteins und O. Spenglers: Über den „primären Sprachspielen“ als den Ausdrucks- und Eindrucksspielen „primitiven Benehmens" (vgl. $Z, \S 545$ ) erheben sich die Sublimationen der „sekundären Sprachspiele“" wie z. B. der Namengebung als der „größte[n] Wendung in der. Geschichte der menschlichen Seele“ $(U A, 723){ }^{23} \mathrm{Da}$ hingehend tendiert L. Wittgenstein dahin, solche „sekundäre Sprachspiele“ als mißverstandene oder mißbrauchte „primäre“ zu detegieren, bzw. „die Wörter von ihrer metaphysischen, wieder auf ihre alltägliche Verwendung“" $(P U, \S 116)$ zurückzuführen. Mit O. Spengler zu sprechen, neigt er dazu, in Sprachkultur und Zivilisation, den vorkulturellen Kern in der Naturgeschichte bzw. im „primitiven Benehmen“ $(Z, \S 54)$ des Menschen sichtbar zu machen.

\section{$* * *$}

Zum Schluß möchte ich die Frage stellen: War L. Wittgenstein von O. Spenglers Sprachphilosophie beeinflußt, oder haben wir es wie häufig in der Ideengeschichte hier nur mit einer quasi-spenglerschen Gleichzeitigkeit (vgl. $U A, 150 \mathrm{f}$.) ohne unmittelbare Beeinflussung zu tun, also nur mit einer Koinzidenz? ${ }^{24} \mathrm{Da}$ L. Wittgenstein von $\mathrm{O}$. Spengler beeinflu $\beta t$ ist, wird von ihm explizit erwähnt (vgl. $V B, 43$ ). Dagegen werden andere ihm bekannte Sprachphilosophen der zwanziger Jahre wie z. B. C. K. Ogden und I. A. Richards in der Liste nicht erwähnt (vgl. $V B, 43$ ). Daß L. Wittgenstein aber nicht nur im ersten Band Gestalt und Wirklichkeit, sondern auch im zweiten Band Welthistorische Perspektiven gelesen hat, ist ebenfalls beweisbar. In den Vermischten Bemerkungen schreibt er: „Richtig reiht dabei Spengler Weininger nicht unter die westlichen Philosophen [Denker]“" $(V B, 38)$. O.

23 Der Verfasser ist für diese Unterscheidung zwischen primären und sckundären Sprachspielen einer mündlichen Mitteilung J. Hintikkas verpflichtet.

24 Vgl. zum Gesetz der Koinzidenz bzw. der Serie die interessanten Bemerkungen Hohls 1986, $390 \mathrm{f}$. 
Weininger gehört nämlich für $O$. Spengler wie dic jüdischen Denker Spinoza und S. Maimon zu den „drei Heilige[n] im Sinne des morgenlïndischen Sufismus“ $(U A, 956), \ldots$. , die man als solche nur erkennt, wenn man durch den Anflug abendländischer Denkformen hindurchzusehen vermag" $(U A, 956)$. Hat $\mathrm{L}$. Wittgenstein aber schon im dritten Kapitel des zweiten Bandes Probleme der arabischen Kultur gelesen, so ist zu vermuten, daß er auch im zweiten Kapitel Städte und Völker gelesen hat, worin sich der Abschnitt über Völker, Rassen, Sprachen (vgl. $U A, 688-745$ ) findet. Dieser mußte ihn mindestens so sehr wie Probleme der arabischen Kultur interessieren. Da ich allerdings kein wörtliches Zitat gefunden habe, so ist das bis dahin nur eine Vermutung, aber kein Beweis.

Sie läßt sich jedoch durch ein Indiz stützen. Nach N. Malcolms bekannter Anekdote soll eine bestimmte Geste P. Sraffas „..., wie sie den Neapolitanern geläufig ist, wenn sie so etwas wie Abscheu oder Verachtung ausdrücken wollen: $\mathrm{Er}$ fuhr mit den Fingerspitzen der nach außen gekehrten Hand über die Unterseite des Kinns ..." 25 und die Frage ,, Was ist die logische Form davon?" " 26 in L. Wittgenstein das Gefühl hervorgerufen haben, es sei absurd, darauf zu beharren, daß ein Satz und das, was er darstellt, dieselbe ,Form“ haben müssen: „Dadurch löste er sich von der Auffassung, der Satz müsse buchstäblich ein ,Bild“ der Wirklichkeit sein, die er darstellt."27 Nun gibt es im Abschnitt über Völker, Rassen, Sprachen folgende Anmerkung: „Die heutigen Gebärdensprachen (Delbrück, Grundfragen d. Sprachforschung, S. 49 ff., mit dem Hinweis auf das Werk von Jorio über die Gesten der Neapolitaner) setzen sämtlich die Wortsprache voraus ...“ $\left(U A, 724^{1}\right)$. Diese Ansicht wäre auch konsistent mit der Auffassung des Tractatus vom Primat der Wortsprache. ${ }^{28}$ Dahingegen setzt in O. Spenglers morphologischer Betrachtung die Wortsprache die Gebärdensprache voraus. Das Werk A. de Jorios La mimica degli antichi investigata nel gestire napoletano zeigt aber folgende, in der Wittgensteinliteratur noch unbekannte Abbildung von P. Sraffas Geste

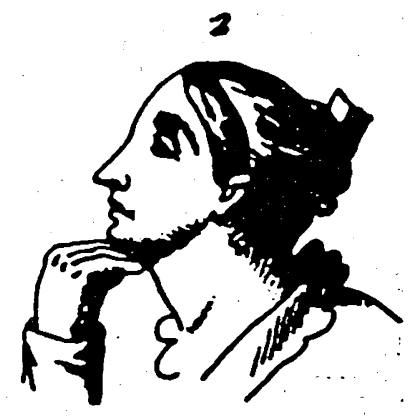

und enthält folgenden Kommentar:

Malcolm 1987, 94.

Malcolm 1987, 94.

Malcolm 1987, $94 \mathrm{f}$.

28 Darauf macht richtig Lüdeking 1988, 413 aufmerksam, ohne Hinweis auf Spengler und De Jorio. 
„Estremi esterni delle dita puntate sotto al mento, e spinte con violenza in fuori (v. tav. 21. n.2). Dopo l'anzidetto si comprende chiaro, come con simile atteggiamento il mimico vi dinota che egli vuole allontanare la sua testa da ciò che gli si offre o propone, perchè non gli aggrada. Per eseguirlo presto e con forza, riccorre alla mano, acciò o con gli estremi esterni delle dita, o con le sole punte delle unghia, faccia atto di spingere al più lontano, che può, la sua testa; la quale in questo caso si prende anche per l'intera persona." 29 .

Subsumiert aber wird diese Geste unter der Rubrik „NEGATIVA, NO“:

"Si può dir di no col gestire, ed in diversi modi: Con gli occhi, con la testa, con la testa e le mani, con le mani sole, e con tutto il corpo, o col semplice trarre in su le spalle, infossando il collo. Per quello che riguarda lo spirito del gesto è anche vario, cioè Negativa con indifferenza, con impegno, con sorpresa, con isdegno od orrore, o finalmente con ironia; la quale, come si è detto, può accoppiarsi a tutti i gesti.“ 30

Ich behaupte nicht, daß L. Wittgenstein dieses im übrigen hochinteressante und gut gearbeitete Werk gelesen hat. Eine Entscheidung darüber könnte nur die Bibliothek L. Wittgensteins, die wohl kaum mehr vorhandenen Ausleihzettel der von ihm benutzten Bibliotheken oder ein Eintrag aus dem noch nicht publizierten Nachla $B$ bringen. Meine Vermutung ist nur die, daß die Geste P. Sraffas schwerlich auf L. Wittgenstein einen so nachdrücklichen Eindruck gemacht hätte, wenn sie nicht an die fundamentale Bedeutung der Gestensprache für die Wortsprache angeklungen hätte, wie sie O. Spengler eindrücklich dargestellt und wie sie L. Wittgenstein aus seiner Spenglerlektüre bekannt gewesen sein konnte. ${ }^{31}$ Die „Grammatik" ${ }^{32}$ von P. Sraffas Geste läßt sich nämlich mit der Bildtheorie des Satzes nicht mehr verstehen:

(a) Während die doppelte Negation eines verneinenden Satzes den Satz bejaht (vgl. TLP, 5.44), so würde eine Wiederholung dieser verneinenden Geste die Verneinung nicht aufheben, sondern verstärken, d.h. die logische Konstante der Negation ist auf P. Sraffas Geste nicht anwendbar. Deshalb ist wohl P. Sraffas Geste nicht als logische Partikel, sondern als Satz mit propositionalem Gehalt zu verstehen. ${ }^{33}$

29 De Jorio 1832, 224.

30 De Jorio 1832, 222.

31 Vgl. dazu die Bemerkung Weiningers 1903: „Der psychische Bestand des Menschen mit einem System von eng einander benachbarten Glocken verglichen, so gilt ...; für das Genie, daß eine einzige, angeschlagen, gewaltig ausschwingt, nicht leise tönt, sondern voll, das ganze System mitbewegt, ..." (156).

32 Wir folgen hier der Wiedergabe der Episode v. Wrights nach Malcolm 1986, 952: „G. H. von Wright teilt mir mit, daß Wittgenstein ihm diese Begebenheit in etwas anderer Weise erzählt habe: Die Streitfrage war, nach Wittgenstein, ob jeder Satz eine ,Grammatik' haben müsse, und Sraffa fragte Wittgenstein, was die ,Grammatik' jener Geste sei. Als Wittgenstein diese Begebenheit von Wright beschrieb, gebrauchte er die Formulierung, logische Form' oder ,logische Mannigfaltigkeit" nicht."

33 Darauf hat Lüdeking 1988, 414, aufmerksam gemacht. Die Abbildung bestätigt die Vermutung, daß es sich hier um die Geste der Negation handelt. 
(b) Die Bildtheoric des Satzes kann angesichts der ,.scheinbare/n] Unregelmäßighaifin" (vgl. TL.P, 4.013) nur deshalb aufrechterhalten werden (vgl. TL.P, 4.021), weil der Sitz. eine Wahrheitsfunktion der Elementarsätze ist (vgl. TLP, 5). Erst dic Eilementarsätze und deren Kombinationen durch die Wahrheitsfunktion sind dann in „buchstïblichem" Sinne von „aRb“ (vgl. TLP, 4.012) Bilder der Tatsachen, weil erst die Elementarsätze mit den elementaren Tatsachen „,isomorph“ sind. ${ }^{34}$ Wenn uun die Elementarsätze mit den elementaren Tatsachen ,isomorph“ sind, so auch deren Kombinationen durch die Wahrheitsfunktion. ${ }^{35}$ Deren allgemeine Form lautet: „Dic allgemeine Form der Wahrheitsfunktion ist: $[\overline{\mathrm{p}}, \xi, \mathrm{N}(\xi)]$. Dies ist die allgemeine Form des Satzes.“ (TLP, 6.) Diese ,allgemeine Form des Satzes“ enthält aber mit der einzigen logischen Konstante $\mathrm{N}$ die in der Gestensprache nicht mehr anwendbare logische Konstante der Negation: „N $(\xi)$ ist die Negation sämtlicher Werte der Satzvariablen" (TLP, 5.502 b.) Nun ist es aber absurd anzunehmen, daß P. Sraffas Geste in „Elementargesten“ und deren Kombinationen durch die Wahrheitsfunktion analysiert werden könnte, die dann in „buchstäblichem“ Sinne mit den elementaren Tatsachen „isomorph“ sind. Denn diese Wahrheitsfunktion enthält ja die iterierbare logische Konstante der Negation.

(c) Während die Analyse von Sätzen in Elementarsätze wegen der Einfachheit der in den elementaren Tatsachen „verketteten“ Gegenstände die kontextunabhängige „Bestimmtheit des Sinnes“ dieser Sätze garantiert (vgl. TLP, 3.23), so wäre es absurd anzunehmen, daß der Sinn von P. Sraffas Geste je durch eine Analyse kontextunabhängig und absolut bestimmt gemacht werden kann, weil sie wie ein Satz im Tractatus im Prinzip quasi „von Natur" absolut bestimmt ist. In den $P U$ führt dann L. Wittgenstein die Negation auf ihren gestischen Gehalt zurück: „Die Negation, könnte man sagen, ist eine ausschließende, abweisende Gebärde. Aber eine solche Gebärde verwenden wir in sehr verschiedenen Fällen!“ $(P U, \S 550)$. Erst diese verschiedenen Fälle der Verwendung, d.h. etwa der jeweilige Kontext in den neapolitanischen Lebensformen, ergeben den jeweils kontextabhängigen und relativ bestimmten Sinn der Gebärde. Doch wiewohl es so absurd erscheint, P. Sraffas Geste als Satz im Sinne des Tractatus zu verstehen, so können wir uns doch mit solchen Gebärden wie mit einem Satz mit propositionalem Gehalt verständigen. Dafür bieten A. de Jorios und $\mathrm{O}$. Spenglers Werk reichliche Evidenz. Dies ist mit der Bildtheorie des Tractatus nicht erklärbar. P. Sraffas Geste erscheint so als eine der Anomalien (im Kuhnschen Sinne) für die Tractatustheorie.

Alle Reminiszenzen an O. Spengler in den Vermischten Bemerkungen stammen übrigens mit einer Ausnahme (vgl. $V B, 57)$ aus dem Jahre 1931. 1931 hat O. Spengler auch das Werk Der Mensch und die Technik publiziert. L. Wittgenstein konnte also 1931 nicht nur den Untergang, sondern auch dieses Buch gelesen haben. 1931 dürfte auch die von N. Malcolm erwähnte Episode mit P. Sraffa stattgefunden haben. ${ }^{36}{ }^{\prime} 1931$

\footnotetext{
34 Vgl. Stenius 1969, 121 -132, zum Begriff der Isomorphie.

$35 \mathrm{Vgl}$. dazu die erhellenden Bemerkungen von Hintikka 1988, $382 \mathrm{f}$.

36 So die Konjektur Lüdekings 1988, 414.
} 
aber hielt L. Wittgenstein noch an der Bildtheorie der Sprache fest, wie aus Wittgenstein's Lectures, Cambridge, 1930 - 1932 hervorgeht: "A proposition is a fact which is a picture of another fact". $.37 \mathrm{Die}$ Abkehr von oder besser die Modifikation der Bildtheorie der Sprache durch die Sprachspiele, die erst die Sprache auf die Welt beziehen, ${ }^{38}$ dürfte so erst nach dem Jahre 1931 stattgefunden haben, d. h. also nach der wohl mit der Spenglerlektüre ungefähr gleichzeitigen Episode mit P. Sraffa. Das ist freilich kein Beweis dafür, daß L. Wittgenstein O. Spenglers Sprachphilosophie oder gar das Werk A. de Jorios gelesen hat. Doch die Indizien deuten darauf hin, daß L. Wittgenstein wenigstens O. Spenglers Sprachphilosophie gelesen haben könnte. O. Spenglers Philosophie enthält ja mit ihrer Betonung der Gestensprache u. a. ähnliche Anomalien für die Bildtheorie des Tractatus wie P. Sraffas Geste und verweist mit dem Hinweis auf das Werk A. de Jorios zumindest implizit auch auf die Geste P. Sraffas. Freilich finden wir keine wörtliche Übernahme. L. Wittgenstein hat - mit seinen eigenen Worten - die Spenglersche Gedankenbewegung nur sogleich leidenschaftlich zu seinem Klärungswerk aufgegriffen (vgl. VB, 43). Rezeption heißt hier Transplantation in einen anderen Organismus. Die Originalität von L. Wittgensteins intellektuellem Organismus aber wäre wie ihm die Originalität S. Freuds nicht eine des „Samens“, sondern des „Bodens“ (vgl. VB, 75); erst recht, wenn das Samenkorn O. Spenglers wie das Samenkorn J. Breuers bei S. Freud „nur ganz winzig“ gewesen sein mag (vgl. $V B, 75)$. Doch hat L. Wittgensteins „Boden“ das Spenglersche Samenkorn ,anders wachsen“ $(V B, 75)$ lassen, als es wohl ,in irgend einem anderen Boden“ $(V B, 75)$ gewachsen wäre.

\section{Erwähnte Literatur}

Baker, G.P. and Hacker, P. M.S., Wittgenstein, Understanding and Meaning, An analytical commentary on the Philosophical Investigations, I, Oxford 1980.

Bloor, D., Wittgenstein, A Social Theory of Knowledge, London 1983.

Hacker, P. M.S., siehe unter Baker, G.P.

Haller, R., War Wittgenstein von Spengler beeinflußt?, in Fragen zu Wittgenstein und Aufsätze zur Österreichischen Philosophie, hg.v. R. Haller, Amsterdam 1986, (Studien zur Österreichischen Philosophie, 10), 170-186.

37 Lee $1980,85$.

$38 \mathrm{Vgl}$. Hintikka/Hintikka 1987, 225-232, $239^{20}$, die plausibel machen können, daß L. Wittgenstein dic Bildtheorie der Sprache nicht schlechthin aufgegeben hat.

15 Arch. Gesch. Philosophie Bd. 73 
Hilmy, St. S., The Later Wittgenstein. The Eimergence of a New Philosophical Method, Oxford 1987.

llintikka, M. and J., Investigating Wittgenstein, Oxford/New York 1987.

llintikka, J., „Die Wende der Philosophie": Wittgenstein's New Logic, in Philosophy of Law. Politics, and Society. Proceedings of the 12th International Wittgenstein Symposium. 7th to 14th August 1987, Kirchberg am Wechsel (Austria). Selected Papers, Ed. by O. Weinberger, P. Koller and A. Schramm, Vienna 1988, $380-$ 396.

Hohl, L., Von den hereinbrechenden Rändern, Nachnotizen, Frankfurt a. M. 1986.

Janik, A., Wittgenstein and Weininger, in Essays on Wittgenstein and Weininger, hg. v. R. Haller, Amsterdam 1985, (Studien zur Österreichischen Philosophie, 9), $64-73$.

Jorio de, A., La mimica degli antichi investigata nel gestire napoletano, Napoli 1832, abgedr. Con prefazione di G. Cocciara, Associazione napoletana per i momenti e il paesaggio, Napoli 1964.

Kripke, S. A., Wittgenstein on Rules and Private Language, Oxford 1982.

Lee, D., Wittgenstein's Lectures, Cambridge, 1930-1932, From the Notes of J. King and D. Lee, Ed. by D. Lee, Oxford 1980.

Lüdeking, K.H., Sraffa's Gesture, in Philosophy of Law, Politics, and Society, Proceedings of the 12th International Wittgenstein Symposium, 7th to 14th August 1987, Kirchberg am Wechsel (Austria). Selected Papers, Ed. by O. Weinberger, P. Koller and A. Schramm, Vienna 1988, 413-416.

Malcolm, J., Nothing is hidden, Wittgenstein's Criticism of his Early Thought, Oxford 1987.

Malcolm, J., Erinnerungen an Wittgenstein, Mit einer biographischen Skizze von G. H. v. Wright und Wittgensteins Briefen an N. Malcolm, aus dem Englischen von C. Frank und J. Schulte, Frankfurt a. M. 1987.

McGinn, C., Wittgenstein on Meaning, Oxford 1984.

McGuiness, B., Wittgensteins frühe Jahre, aus dem Englischen v. J. Schulte, Frankfurt a. M. 1988.

Pears, D., The false Prison, A Study of the Development of Wittgenstein's Philosophy, 1, Oxford 1987, 2, Oxford 1988.

Savigny, E. v., Wittgensteins Philosophische Untersuchungen, Ein Kommentar für Leser, 1, Abschnitte 1 bis 315, Frankfurt a. M. 1988.

Smith, B., Weininger und Wittgenstein, in Theoria 5, 1985, 227-237, [Estratto].

Spengler, O., Der Untergang des Abendlandes, Umrisse einer Morphologie der Weltgeschichte, 1, Gestalt und Wirklichkeit, 2, Welthistorische Perspektiven, München 192333-47. Zitiert nach der Ausgabe im Deutschen Taschenbuch Verlag mit Nachwort v. A. M. Koktanek, München $1972,1988^{9}=[U A]$.

Spengler, O., Der Mensch und die Technik, Beitrag zu einer Philosophie des Lebens, München 1931.

Spengler, O., Frühzeit der Weltgeschichte, Fragmente aus dem Nachlaß, unter Ḿitwirkung v. M. Schröter hg. v. A. M. Koktanek, München 1966.

Schulte, J., Chor und Gesetz. Zur „Morphologischen Methode“ bei Goethe und Wittgenstein, in Grazer Philosophische Studien, Internationale Zeitschrift für Analytische Philosophie, hg. v. R. Haller, 21, 1984, 1-32. 
Stenius, E., Wittgensteins Traktat, aus dem Englischen v. W. Bader, Frankfurt a. M. 1969.

Weininger, O., Geschlecht und Charakter. Eine prinzipielle Untersuchung, im Anhang Weiningers Tagebuch, Briefe A. Strindbergs sowie Beiträge aus heutiger Sicht v. A. Stopczyk, G. Dischner u. R. Calasso, München 1980, Nachdruck der 1. Auflage, Wien 1903.

Wittgenstein, L., Tractatus logico-philosophicus in Schriften 1, Frankfurt a. M. 1969 $=[T L P]$.

Wittgenstein, L., Philosophische Untersuchungen in Schriften 1, Frankfurt a. M. 1969 $=[P U]$.

Wittgenstein, L., Philosophische Bemerkungen, aus dem Nachlaß hg. v. R. Rhees in Schriften 2, Frankfurt a. M. $1969=[P R]$.

Wittgenstein, L., Philosophische Grammatik, hg. v. R. Rhees, in Schriften 4, Frankfurt a. M. $1969=[P G]$.

Wittgenstein, L., Das Blaue Buch - Das Braune Buch, hg. v. R. Rhees in Schriften 5, Frankfurt a. M. $1969=[B L B]-[B R B]$.

Wittgenstein, L., Zettel, hg. v. G.E.M. Anscombe und G.H. v. Wright, in Schriften 5, Frankfurt a. M. $1969=[Z]$.

Wittgenstein, L., Über Gewißheit, hg. v. G.E.M. Anscombe und G. H. v. Wright, Frankfurt a. M. $1971=[\ddot{U} G]$.

Wittgenstein, L., Vermischte Bemerkungen. Eine Auswahl aus dem Nachlaß, hg. v. G. H. v. Wright. Unter Mitarbeit v. H. Nyman, Frankfurt a. M. $1977=[V B]$. Wittgenstein, L., Bemerkungen über Frazers „Golden Bough" in Ludwig Wittgenstein, Vortrag über Ethik und andere kleine Schriften, hg. und übers. v. J. Schulte, Frankfurt a. M. 1989, 29-46 = $[B F G B]$.

Wright, G.H. v., Wittgenstein, aus dem Englischen v. J. Schulte, Frankfurt a. M. 1982. 